\title{
sciendo
}

DOI: 10.1515/aon-2019-0007

\section{PERFORMANCE ANALYSIS OF GPS/BDS INTEGRATED PRECISE POSITIONING SYSTEM CONSIDERING VISIBILITY OF SATELLITES}

\author{
Jae Hee Noh \\ Department of Electronics Engineering \\ Chungnam National University \\ 99, Deahak-ro, Yuseong-Gu, Daejeon, Republic of Korea \\ jhnoh3555@cnu.ac.kr \\ Gwang Hee Jo \\ Department of Electronics Engineering \\ Chungnam National University \\ 99, Deahak-ro, Yuseong-Gu, Daejeon, Republic of Korea \\ j_ghee@cnu.ac.kr \\ Deok Won Lim \\ Satellite Navigation Team \\ Korea Aerospace Research Institute \\ 169-84, Gwahak-ro, Yuseong-Gu, Daejeon, Republic of Korea \\ dwlim@kari.re.kr \\ Jin Hyuk Lee \\ Department of Electronics Engineering \\ Chungnam National University \\ 99, Deahak-ro, Yuseong-Gu, Daejeon, Republic of Korea \\ jh_lee@cnu.ac.kr \\ Sun Yong Lee \\ Navcours Co., Ltd. \\ 66-6, Techno2-ro, Yuseong-Gu, Daejeon, Republic of Korea \\ sy_lee@cnu.ac.kr \\ Sang Jeong Lee \\ Department of Electronics Engineering \\ Chungnam National University \\ 99, Deahak-ro, Yuseong-Gu, Daejeon, Republic of Korea \\ eesj1@cnu.ac.kr
}

\begin{abstract}
Research on precise positioning is being actively carried out to provide accurate position information for land transportation. The most significant problem when performing precise positioning in urban canyon is the degradation of performance due to the lack of visible satellites. Prior to open service of BDS, most of the studies on positioning were focused on using GPS/GLONASS integrated navigation system. Since BDS began open service, studies using GPS/BDS have been actively performed in the Asia-Pacific region as it became possible to acquire enough available BDS satellites. The average number of visible satellites in Korea is 9 for GPS and 14 for BDS. In this paper, we analyze the availability of precise positioning using BDS in urban canyon. To do this, we simulate the urban canyon environment by applying the mask to the azimuth and the elevation. We analyze the positioning accuracy using two simulation scenarios. From the results, it is
\end{abstract}


shown that the accuracy of precise positioning in the case where the satellites in the east-west direction are blocked is lowered than that in the case where the satellites in the south-north direction are blocked for the same elevation mask angle. This result comes from the fact that the PDOP increases when the satellites are blocked in the east-west direction. Also, it can be confirmed that the GPS/BDS integrated positioning is available for the high mask angle while the GPS-only positioning is not possible continuously.

Keywords - BDS, Precise Positioning, Urban Canyon

\section{INDRUCTION}

In recent years, Intelligent Transportation Systems (ITS) to provide convenient services to the users of the traffic system has actively studied around the world. In order to provide advanced service, land transportation requires a position accuracy of less than $50 \mathrm{~cm}$ (2DRMS) with which it can be possible to distinguish lanes[1]. Research on precise positioning by using the satellite navigation system is being actively carried out to provide accurate position information for land transportation. In urban canyon environment, high-rise bridges and high-rise buildings have the problem of blocking GNSS signal. The blocking of the GNSS signal affects the number of visible satellites used for positioning. This leads to a lack of the number of visible satellites, which causes the degradation of the performance of positioning to decrease. Prior to the development of other satellite navigation systems, the study of precise positioning were performed using mainly GPS. After other satellite navigation system such as GLONASS and BDS were developed and provided the global service, the precise positioning has been performed based on multi-GNSS. In case of performing Multi-GNSS precise positioning in the urban canyon, enough visible satellites can be acquired. For that reason, the performance of positioning is improved because the number of code measurements and carrier phase measurements increases. Therefore, studies on multi-GNSS precise positioning are needed to obtain the accurate information of user location in urban canyon. Prior to the open service of BDS, most of the studies on positioning were focused on using GPS/GLONASS integrated navigation system. However, since BDS began open service, studies using GPS/BDS integrated system have been actively performed in the Asia-Pacific region[2][3]. BDS adopts the same CDMA method as GPS, which makes it easier to implement multiple satellite navigation systems than GLONASS. In the open sky, an average of 9 GPS satellites and 14 BDS satellites could be observed for 24 hours. From this, it can be expected that the number of visible satellite of BDS is more observable than that of GPS in Korea[4].

In this paper, we analyze the availability of precise positioning using BDS in urban canyon environment. To do this, we simulate the urban canyon by applying the mask angle to the azimuth and elevation. For this purpose, Chapter 2 describes the integrated GPS/BDS precise positioning technique. In Chapter 3, we perform the simulation by applying the mask angle to analyze the availability of precise positioning using BDS in urban canyon. And, we analyze the precision positioning performance using two simulation scenario. As a result, we confirmed that the precise positioning availability an accuracy are improved by using an GPS/BDS integrated system.

\section{GPS/BDS PRECISE POSITIONING}

\section{A. GPS/BDS Precise Positioning Technique}

The precise positioning is a relative positioning technique that uses both the code measurement and the carrier phase measurement. By using the carrier phase measurement during the positioning, it is possible to estimate precise positioning with the accuracy of cm-level. The double differenced the code measurement and the carrier phase measurement for each system can be expressed as in Eqs. (1-4). The double differenced code measurement of GPS is

$$
\nabla \Delta P_{u r, G P S}^{i j}=\nabla \Delta \rho_{u r, G P S}^{i j}+\nabla \Delta \varepsilon_{P(u r, G P S)}^{i j}
$$


The double differenced code measurement of BDS is

$$
\nabla \Delta P_{u r, B D S}^{i j}=\nabla \Delta \rho_{u r, B D S}^{i j}+\nabla \Delta \varepsilon_{P(u r, B D S)}^{i j}
$$

where $\varepsilon$ is the measurement noise, $\rho$ is the geometric distance between the satellite and the receiver. $u, r$ refer to the user's receiver and the receiver of reference station, respectively. i, j are i-th and $\mathrm{j}$-th satellites, respectively.

The double differenced carrier phase measurement of GPS is

$$
\lambda_{G P S} \nabla \Delta \phi_{u r, G P S}^{i j}=\nabla \Delta \rho_{u r, G P S}^{i j}+\lambda_{G P S} \nabla \Delta \widetilde{N}_{u r, G P S}^{i j}+\nabla \Delta \varepsilon_{\phi(u r, G P S)}^{i j} .
$$

The double differenced carrier phase measurement of BDS is

$$
\lambda_{B D S} \nabla \Delta \phi_{u r, B D S}^{i j}=\nabla \Delta \rho_{u r, B D S}^{i j}+\lambda_{B D S} \nabla \Delta \widetilde{N}_{u r, B D S}^{i j}+\nabla \Delta \varepsilon_{\phi(u r, B D S)}^{i j}
$$

where $\lambda$ is the length of one wavelength of the carrier phase measurement of GPS L1 signal and BDS B1 signal. $N$ refers to the integer ambiguity in the carrier phase measurement of GPS and BDS. The conceptual structure that linearizes the double differenced measurements in the short-baseline can be defined as

$$
y=\left[\begin{array}{l}
y_{P} \\
y_{\phi}
\end{array}\right]=\left[\begin{array}{ll}
B & 0 \\
B & { }_{A}
\end{array}\right]\left[\begin{array}{l}
b \\
a
\end{array}\right]+\left[\begin{array}{l}
\varepsilon_{P} \\
\varepsilon_{\phi}
\end{array}\right]
$$

where $\mathrm{y}$ is defined as $y=\left[\begin{array}{lllllll}y_{1, P} & y_{2, P} & \ldots & y_{n, P} & y_{1, \phi} & \ldots & y_{n, \phi}\end{array}\right] . \mathrm{P}$ is the double differenced code measurement and $\phi$ is the double differenced carrier phase measurement. $b$ is a 3-dimensional position vector, $\mathrm{a}$ is $a$ double difference integer ambiguity vector, and $\mathrm{B}$ and $\mathrm{A}$ represent coefficient matrices of $\mathrm{b}$ and $\mathrm{a}$, respectively. If the equation defined in Eq.(5) is described by the equation for the GPS / BDS integrated system, the equation is

$$
y=\left[\begin{array}{l}
y_{P, G P S} \\
y_{\phi, G P S} \\
y_{P, B D S} \\
y_{\phi, B D S}
\end{array}\right]=\left[\begin{array}{ccc}
B_{G P S} & 0 & 0 \\
B_{G P S} & A_{G P S} & 0 \\
B_{B D S} & 0 & 0 \\
B_{R n S} & 0 & A_{R D S}
\end{array}\right]\left[\begin{array}{c}
b \\
a_{G P S} \\
a_{B D S}
\end{array}\right]+\left[\begin{array}{c}
\varepsilon_{P, G P S} \\
\varepsilon_{\phi, G P S} \\
\varepsilon_{P, B D S} \\
\varepsilon_{\phi, B D S}
\end{array}\right] .
$$

When two or more GNSS integrated, the 3-dimensional position vector should include all of the clock error terms for each GNSS[5].

B. Satellite's visibility

Figs. 1-2 show the number of visible satellite of GPS and BDS for 24 hours in open sky. The mask angle of elevation is $10^{\circ}$. 


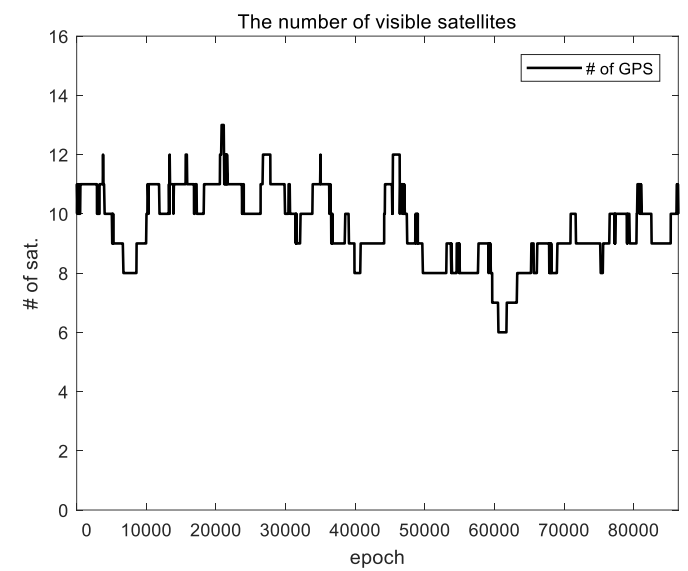

Fig. 1. The number of visible satellite of GPS for 24 hours

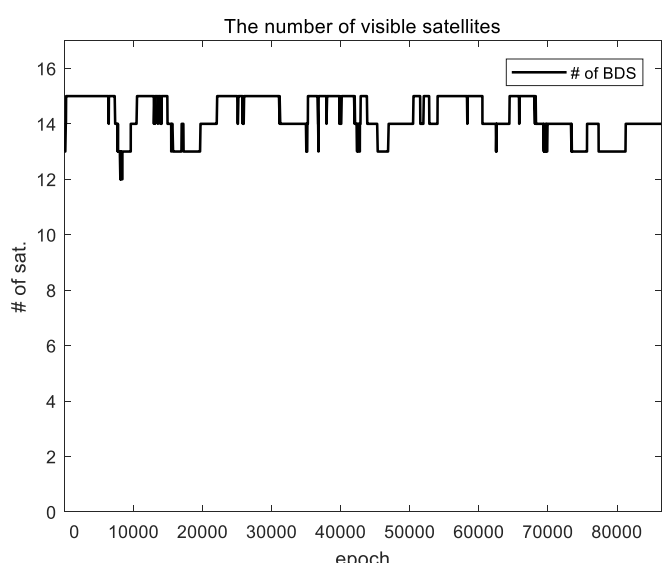

Fig. 2. The number of visible satellite of BDS for 24 hours

Where the $\mathrm{x}$-axis refers to the time and the $\mathrm{y}$-axis refers to the number of visible satellites. According to the results, an average of 9 GPS satellites and 14 BDS satellites could be observed for 24 hours.

\section{Perfromance Analysis of Precise Positioning}

\section{A. Scenario for simulation}

We applied a mask angle to the azimuth to reflect the effect of the satellite signal being blocked according to the direction in which the building was located. We applied the mask angle to elevation to reflect the effect of the GNSS signal being blocked according to the height of the building. The scenario of the precise positioning of performance analysis set in this paper can be roughly classified into two types. Scenario 1 simulates an environment in which the satellite is blocked in the north-south direction. Scenario 2 simulates an environment in which the satellite is blocked in the east-west direction. Fig. 3 shows an example of a mask angle setting for describing a case where a signal is blocked in urban canyon environment. The mask angle of the azimuth was set at $90^{\circ}$. 


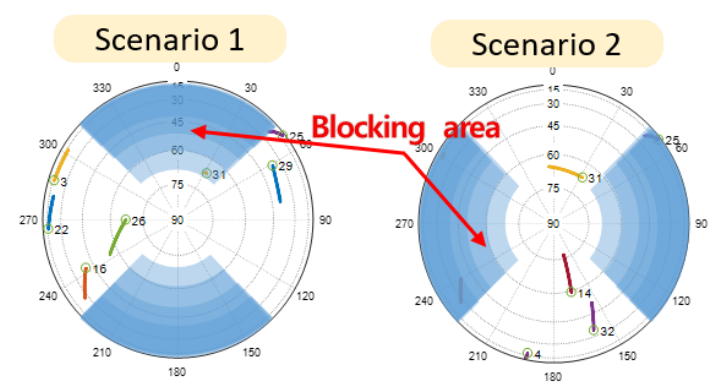

Fig. 1. Example of the blocking scenario

TABLE I shows values of the mask angle of the elevation angle at which the satellite is blocked by the building height. The building height calculated in TABLE I assumes that the user is $25 \mathrm{~m}$ away from the building and the height of one floor is $3 \mathrm{~m}$. The example of this assumption is shown in Fig. 4.

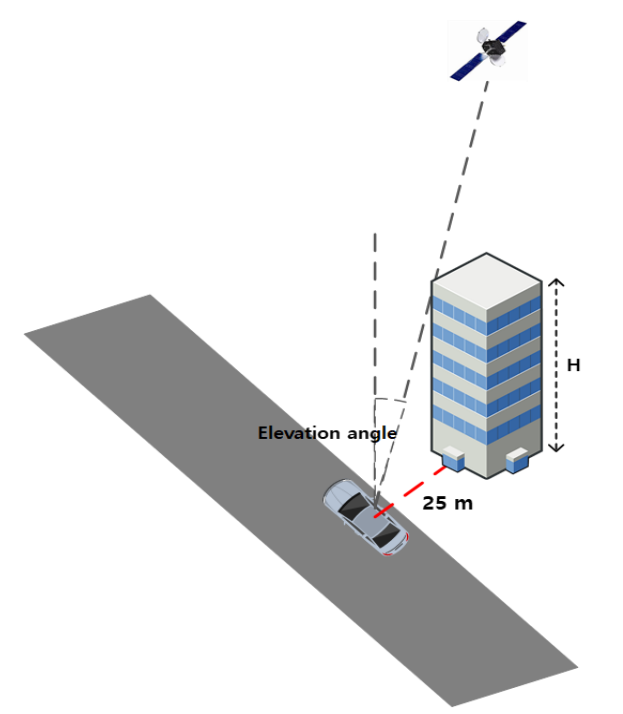

Fig. 2. Example for calculating elevation mask angle

TABLE I. THE LIST OF SCENARIO

\begin{tabular}{|c|c|c|c|c|}
\hline \multicolumn{2}{|c|}{ Scenario 1 } & \multicolumn{2}{c|}{ Scenario 2 } & \multirow{2}{*}{ Description } \\
\cline { 1 - 4 } \# Case & Elev. mask & \# Case & Elev. mask & \\
\hline $1-1$ & $20^{\circ}$ & $1-1$ & $20^{\circ}$ & 3rd Floor \\
\hline $1-2$ & $30^{\circ}$ & $1-2$ & $30^{\circ}$ & 4th Floor \\
\hline $1-3$ & $40^{\circ}$ & $1-3$ & $40^{\circ}$ & 6th Floor \\
\hline $1-4$ & $50^{\circ}$ & $1-4$ & $50^{\circ}$ & 9th Floor \\
\hline $1-5$ & $60^{\circ}$ & $1-5$ & $60^{\circ}$ & 14th Floor \\
\hline $1-6$ & $70^{\circ}$ & $1-6$ & $70^{\circ}$ & 22th Floor \\
\hline
\end{tabular}

\section{B. Experimental Configuration}

In order to analyze the precise positioning performance for GPS-only and the GPS/ BDS integrated system, this study set a zero-baseline environment in an open sky to collect signals and designed a platform to analyze the performance of precise positioning. Fig. 5 shows the environment for collecting GPS L1 signal and BDS B1 signal for 24 hours (December 4, 2018, 01:30 (UTC+09:00) - December 5, 2018, 01:30 (UTC+09:00)). NovAtel GPS-703-GGG antenna and 2 u-blox EVK-M8T used for signal collecting. 


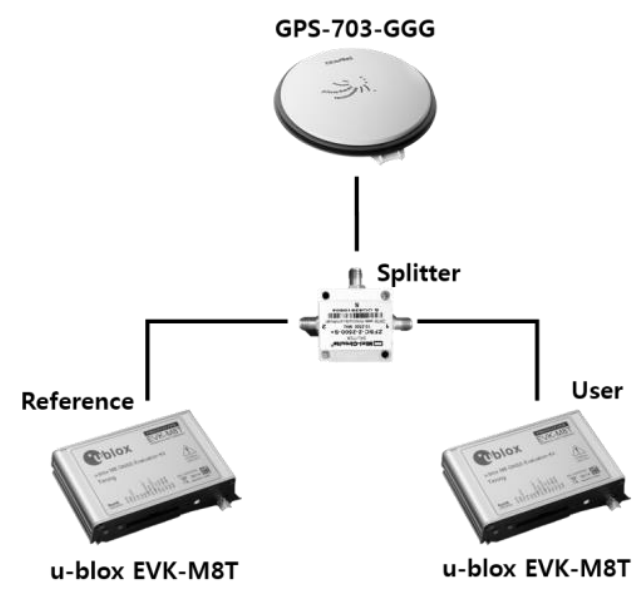

Fig. 3. Configuration for collecting signal

Fig. 6 is a block diagram of the platform designed for precise positioning, where the linearization model is implemented by using the double differenced code and carrier phase measurements obtained in the preprocessing part. This platform estimate the fixed solution, the float solution, and the integer ambiguity.

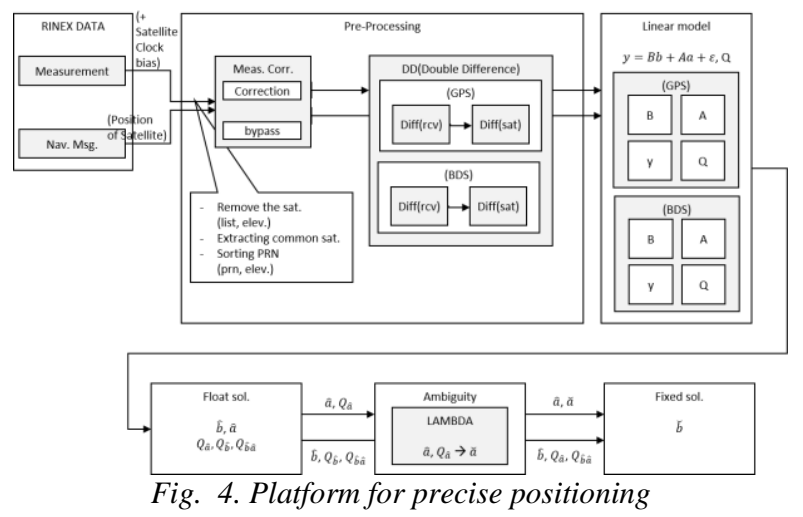

In order to assume an environment where GNSS signals are blocked due to the high buildings in an urban canyon environment, we designed the platform to analyze the performance of precise positioning by applying the mask angle of the azimuth and the elevation.

\section{Pefromance of precise positioning according to scenario}

- Scenario 1

Figs. 7-8 show the result of precise positioning in an environment where the satellite is blocked in the north-south direction and the mask angle of the elevation is $20^{\circ}$. Where the $\mathrm{x}$-axis is the time, and the y-axis refers to the 2DRMS error about the float solution and the fixed solution. In this environment, the probability of estimating the fixed solution within $1 \mathrm{~cm}$ for 24 hours is $99.6 \%$ when performing the GPS-only precise positioning. When performing the GPS/BDS integrated precise positioning, the probability is $99.9 \%$. In an environment where the satellite is blocked in the north-south direction and the mask angle of the elevation is $20^{\circ}$, both the GPS-only and the GPS/BDS integrated system show a high probability of maintaining the precise positioning for 24 hours. 


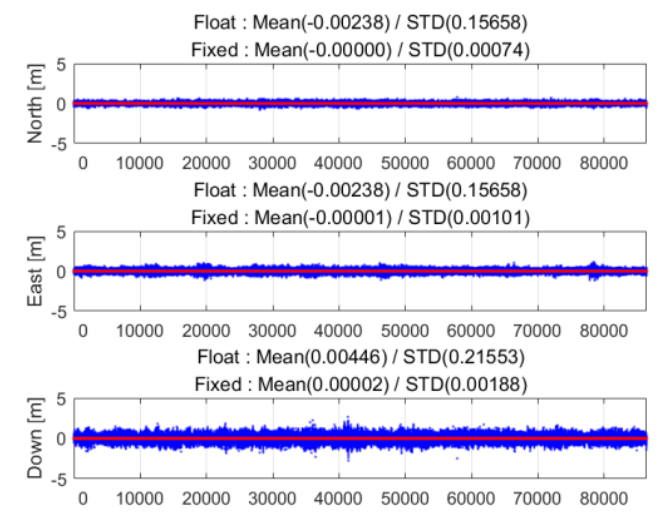

Fig. 5. The result of precise positioning at mask angle $20^{\circ}$ (GPS only)

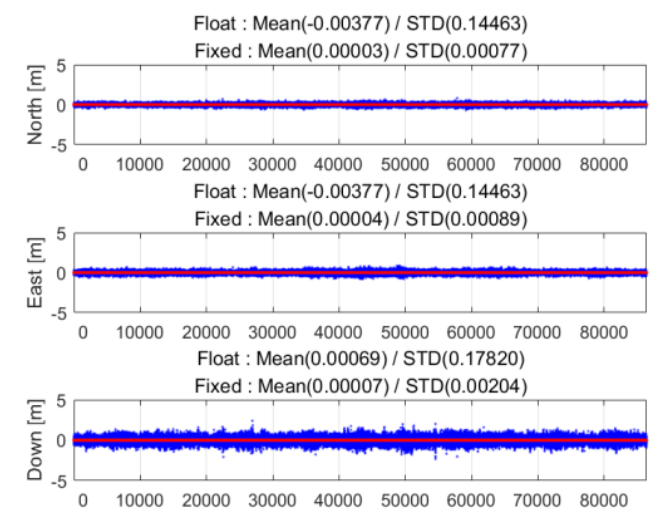

Fig. 6. The result of precise positioning at mask angle $20^{\circ}(G P S+B D S)$

Figs. 9-10 show the result of precise positioning in an environment where the satellite is blocked in the north-south direction and the mask angle of the elevation is $50^{\circ}$. In this environment, the probability of estimating the fixed solution within $1 \mathrm{~cm}$ for 24 hours is $92.0 \%$ when performing the GPS-only precise positioning. When performing the GPS/BDS integrated precise positioning, the probability is $99.9 \%$. In an environment where the satellite is blocked in the north-south direction and the mask angle of the elevation is $50^{\circ}$, both the GPS-only and the GPS/BDS integrated system show a high probability of maintaining the precise positioning for 24 hours.

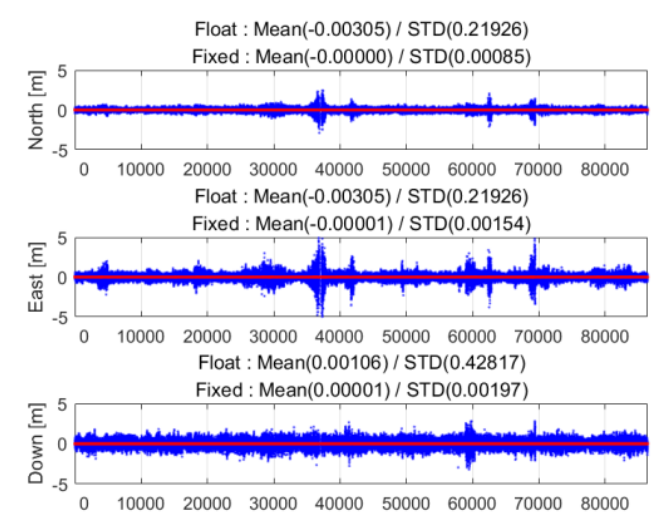

Fig. 7. The result of precise positioning at mask angle $50^{\circ}$ (GPS only) 


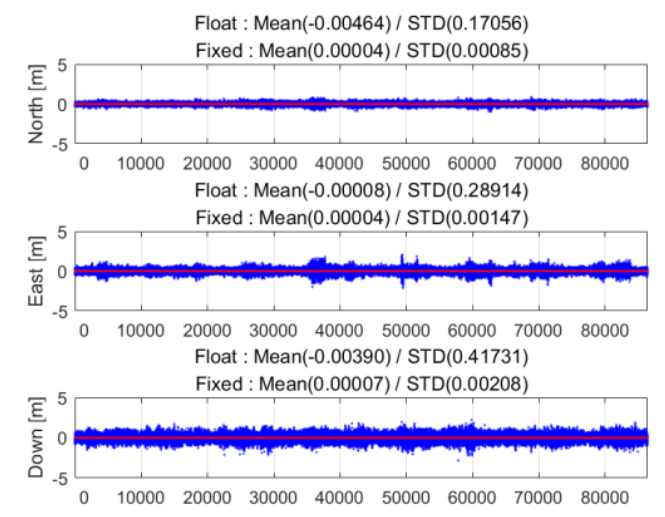

Fig. 8 . The result of precise positioning at mask angle $50^{\circ}(G P S+B D S)$

- Scenario 2

Figs. 11-12 show the result of precise positioning in an environment where the satellite is blocked in the east-west direction and the mask angle of the elevation is $20^{\circ}$. In this environment, the probability of estimating the fixed solution within $1 \mathrm{~cm}$ for 24 hours is $97.9 \%$ when performing the GPS-only precise positioning. When performing the GPS/BDS integrated precise positioning, the probability is $99.6 \%$. In an environment where the satellite is blocked in the east-west direction and the mask angle of the elevation is $20^{\circ}$, both the GPS-only and the GPS/BDS integrated system show a high probability of maintaining the precise positioning for 24 hours.

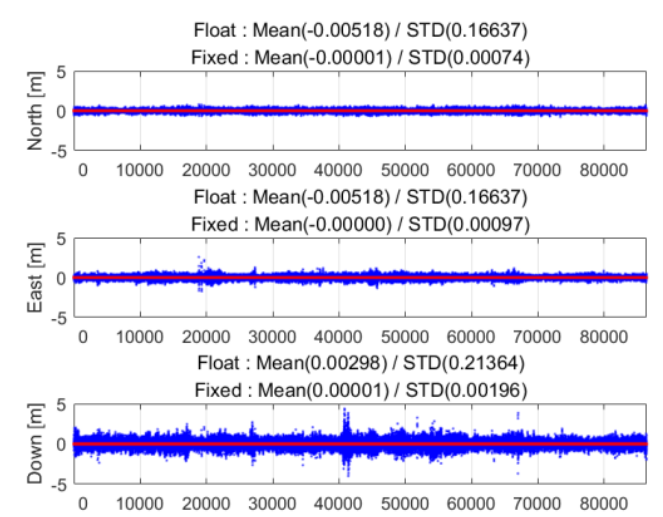

Fig. 9. The result of precise positioning at mask angle $20^{\circ}$ (GPS only)

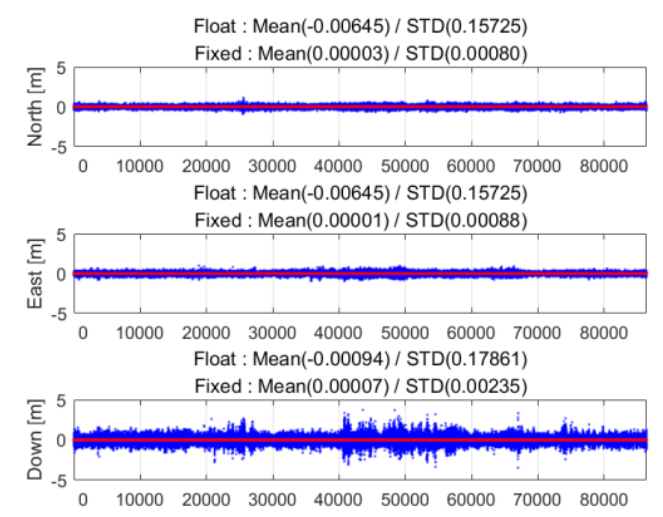

Fig. 10. The result of precise positioning at mask angle $20^{\circ}(G P S+B D S)$

Figs. 13-14 show the result of precise positioning in an environment where the satellite is blocked in the east-west direction and the mask angle of the elevation is $50^{\circ}$. In this environment, the probability of 
estimating the fixed solution within $1 \mathrm{~cm}$ for 24 hours is $51.5 \%$ when performing the GPS-only precise positioning. When performing the GPS/BDS integrated precise positioning, the probability is $89.3 \%$. When the satellite is blocked in the east-west direction, the performance of the GPS-only precise positioning decreases as the mask angle of the elevation increases. However, the GPS/BDS integrated system maintains the high possibility of performing the precise positioning in an environment where the mask angle of elevation is $50^{\circ}$.

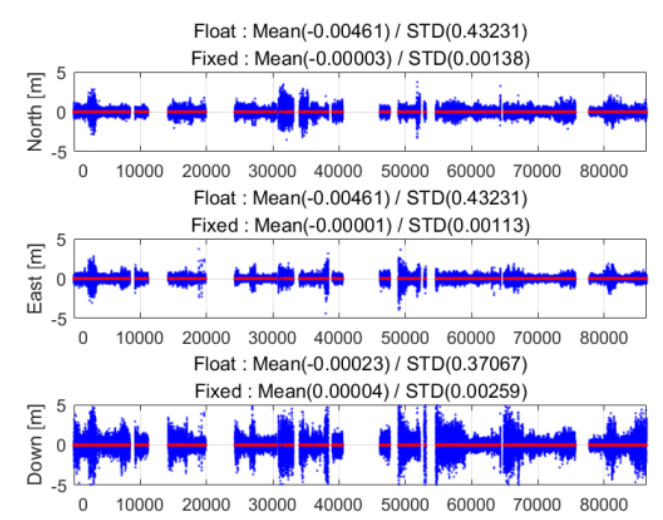

Fig. 11. The result of precise positioning at mask angle $50^{\circ}$ (GPS only)

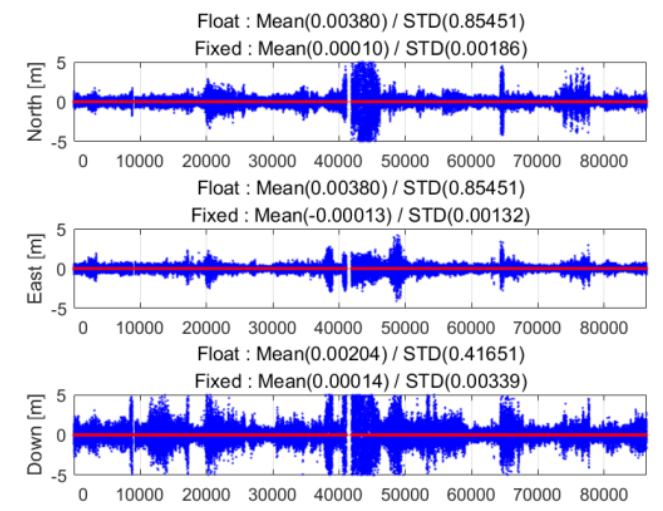

Fig. 12. The result of precise positioning at mask angle $50^{\circ}(G P S+B D S)$

Fig. 15 is a graph showing the possibility of performing the precise positioning within $1 \mathrm{~cm}$ for 24 hours according to the mask angle of the elevation. In the case of scenario 1, the GPS-only and the GPS/BDS integrated system have the high probability of estimating precise positioning in the environment with high mask angle of the elevation. However, in Scenario 2, it is shown that as the mask angle of the elevation increases, the probability of estimating precise positioning decreases sharply in the case of the GPS-only precise positioning. On the contrary, the GPS/BDS integrated system has the high availability in an environment of the high mask angle of the elevation. From the results, it can be seen that the GPS/BDS integrated system has the better performance of the precise positioning than the GPS-only. In addition, when the results of Scenario 1 and Scenario 2 are compared, it can be seen that the performance of PDOP(Position Dilution Of Precision) of Scenario 1 is better. Thus, there is no abrupt change in the performance of the GPSonly precise positioning. 


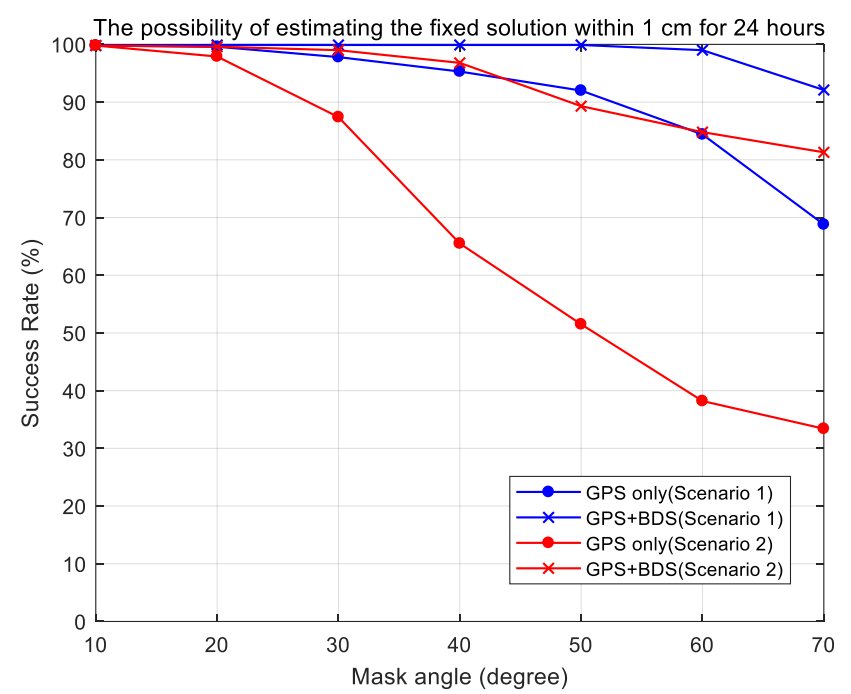

Fig. 13. The possibility of estimating the fixed solution within $1 \mathrm{~cm}$ for 24 hours

\section{CONCLUSION}

In this paper, we analyzed the availability of precise positioning using BDS in urban canyon environment. To do this, we simulated the urban canyon environment. In the environment where the satellite is blocked in the north-south direction, even if the angle of the elevation increases to $50^{\circ}$, the success rate of estimating the fixe solution of $\mathrm{cm}$ level is more than $90 \%$ for both the GPS-only and the GPS/BDS integrated system. In the environment where the satellite is blocked in the east-west direction, the performance of estimating GPS-only precise positioning is rapidly degraded when the mask angle of the elevation is more than $40^{\circ}$. However, the GPS/BDS integrated system showed $81.3 \%$ success rate of estimating the fixed solution of $\mathrm{cm}$ level in the environment where the mask angle of the elevation is $70^{\circ}$. From the results, it was confirmed that it is highly possible to perform precise positioning within $1 \mathrm{~cm}$ even at high mask angle when using the GPS/BDS integrated system compared to when using the GPS-only. This is because the number of BDS visible satellites in Korea is similar to that of GPS, so it can be analyzed as the effect of improving the visibility of satellites by integrating GPS/BDS. The results of this study showed that it is worth using BDS along with GPS for precise positioning in the land transportation. However, it should be mentioned that the visibility of satellites can be not sufficient and multipath can occur in such an environment as urban canyon. Thus, it is necessary to analyze of the relative precise positioning using the GPS and BDS in the actual road environment.

\section{ACKNOWLEDGMENT}

This research was supported by a grant (18TLRP-C113269-03) from Transportation \& Logistics Research Program funded by Ministry of Land, Infrastructure and Transport of Korean government.

\section{REFERENCES}

[1] M. H. Son, E. S. Son, E. S. Lee, M. B. Heo, and G. W. Nam, "Configuration of Network Based GNSS Correction System for Land Transportation Navigation", Journal of the Korean Society for Aviation and Aeronautics, vol. 21, no. 4, pp. 17-26, 2013.

[2] J. Chen, J. Wang, Y. Zhang, S. Yang, Q. Chen, and X. Gong, "Modeling and Assessment of GPS/BDS Combined Precise Point Positioning”, Seonsors, vol. 16, no. 7, pp. 1151-1163, 2016.

[3] S. Zhao, X. Cui, F. Guan, and M. Lu, "A Kalman Filter-Based Short Baseline RTK Algorithm for Single-Frequency Combination of GPS and BDS”, Sonsors, vol. 14, no. 8, pp. 15415-15433, 2014. 
[4] J. H. Noh, S. Y. Lee, D. W. Lim, and S. J. Lee, "Performance Analysis of GPS/BDS Integrated Precise Positioning System Considering Visibility in Urban Environments", Journal of Positioning, Navigation, and Timing, vol. 8, no. 1, pp. 31-40, 2019.

[5] P. Teunissen, and O. Montenbruck, Springer Handbook of Global Navigation Satellite Systems, Cham, Switzerland: Springer, 2017 